\title{
A amígdala e a tênue fronteira entre memória e emoção
}

\author{
Amygdala and the slight boundary between memory and emotion
}

\author{
Fabíola da Silva Albuquerque*; Regina Helena Silva* \\ * Doutora. Professora adjunta, Departamento de Fisiologia, Universidade Federal do Rio Grande do \\ Norte (UFRN), Natal, RN. \\ Este estudo foi realizado no Laboratório de Estudos de Memória, Departamento de Fisiologia, \\ Universidade Federal do Rio Grande do Norte (UFRN), Natal, RN.

\section{Endereço para correspondência}

\section{RESUMO}

Embora os mecanismos neurobiológicos envolvidos na memória para situações aversivas não estejam completamente esclarecidos, o complexo basolateral da amígdala parece exercer um papel fundamental nesse tipo de memória. O presente trabalho tem como objetivo revisar os principais estudos acerca da participação da amígdala na memória e discutir os aspectos teóricos dos modelos utilizados. Foi realizada uma revisão dos estudos em humanos e em modelos animais que visam investigar e discutir a participação da amígdala na memória. Duas hipóteses norteiam as investigações aqui revisadas: 1) a amígdala seria o local dos processos plásticos envolvidos na aquisição e consolidação de informações de conteúdo aversivo e 2) essa estrutura modularia os processos de aquisição e consolidação que ocorreriam em outras estruturas. Os resultados que embasam a primeira hipótese foram obtidos em um modelo de resposta de medo condicionada em ratos, enquanto que a segunda hipótese provém de estudos que utilizam um modelo experimental que envolve aspectos mais declarativos da informação aversiva. Apesar de os trabalhos aqui revisados fornecerem fortes evidências de que a amígdala modula as estruturas relacionadas com a consolidação da memória, novos estudos poderão esclarecer melhor essa relação, principalmente com novos paradigmas de investigação.

Descritores: Amígdala, memória, medo.

\footnotetext{
ABSTRACT

Although the neurobiological events involved with the memory of aversive events are not completely understood, the basolateral amygdala seems to play an important role in this process. The objectives of the present study were to review the main human and animal studies on the role played by the amygdala in memory and to discuss the theoretical aspects of the models used. We reviewed the available studies using human and animal models that investigated and discussed the involvement of amygdala with memory. Current investigation on this issue is mainly based on two hypotheses: 1) the amygdala would be the site of the cellular events that lead to acquisition and consolidation of aversive information and 2) the amygdala would modulate acquisition and consolidation held in other sites within the brain. The first hypothesis is supported by studies conducted in a fear Pavlovian conditioning paradigm, while the second hypothesis is based on studies that use declarative memory procedures. Although the studies we reviewed provide strong evidence that the amygdala modulates brain regions related to memory consolidation, further studies, with new experimental paradigms, could better clarify this relationship.
} 
Keywords: Amygdala, memory, fear.

\section{NTRODUÇÃO}

Medo, prazer, raiva e alegria são significados que atribuímos a conjuntos de sensações subjetivas associadas a reações físicas que se manifestam frente a determinadas situações. Essas e outras emoções refletem a capacidade que os organismos têm de atribuir valências aos eventos do meio, as quais acabam por influenciar seu comportamento. Damásio ${ }^{1}$ propõe que as emoções sejam categorizadas em primárias, ou iniciais (aquelas decorrentes de mecanismos pré-organizados que independem de experiência), e secundárias (as que dependem de aprendizado). As emoções secundárias, segundo esse autor, seriam dependentes de associações sistemáticas entre as emoções primárias e determinados objetos e situações ${ }^{1}$. Uma emoção inata, presente ao nascimento, seria caracterizada como aprendida quando manifestada frente a um objeto ou situação que previamente se associou a uma emoção primária. Dentro do contexto evolutivo, as emoções primárias desempenhariam um papel determinante ao originarem emoções secundárias associadas, por exemplo, a situações de risco de vida ou possibilidade de acasalamento.

O estabelecimento de emoções secundárias depende da capacidade de aprendizagem associativa (uma relação entre estímulos ou entre um estímulo e um comportamento). Enquanto o aprendizado pode ser definido como uma mudança no comportamento que resulta de uma experiência, a memória seria o processo pelo qual aquilo que é aprendido persiste ao longo do tempo e pode ser evocado ${ }^{2}$. Podemos designar a memória necessária a uma emoção secundária como memória emocional.

A memória emocional é utilizada ao se avaliar uma situação ou objeto, permitindo ao organismo reagir de forma adaptativa. Conforme será aqui discutido, a formação da memória emocional é atribuída à atividade de um aglomerado de núcleos subcorticais situados no lobo temporal, que formam o complexo amigdaloide. Coerentemente com o seu papel fundamental na sobrevivência da espécie, tal sistema neural de codificação de memórias com conteúdo emocional parece altamente conservado durante a filogênese dos vertebrados, indo desde o paleocórtex medial, em peixes teleósteos, até a amígdala (do grego amêndoa) em mamíferos ${ }^{3}$.

Apesar de inúmeros estudos apontarem a amígdala como peça chave na formação de memórias com conteúdo emocional, deve-se ressaltar o importante papel dessa estrutura na emoção per se, tanto no que se refere ao reconhecimento e à interpretação das emoções quanto no relativo à expressão fisiológica das respostas emocionais. Nesse sentido, um desafio para os estudos referentes à participação da amígdala na memória seria a separação entre a associação emoção-objeto e a simples expressão da resposta emocional.

O presente trabalho tem como objetivos revisar os principais estudos realizados para investigar a participação da amígdala na memória, discutir os aspectos teóricos dos modelos utilizados com esse fim e analisar as hipóteses propostas para explicar os mecanismos que embasam tal participação.

\section{MÉTODO}

Foi realizada uma pesquisa bibliográfica na base de dados MEDLINE em busca de estudos em humanos e em modelos animais que visam investigar e discutir a participação da amígdala na memória. Os artigos descritos e discutidos na presente revisão foram selecionados com base em sua relevância conceitual ou histórica para o tema proposto, bem como em sua contribuição para o esclarecimento dos mecanismos relacionados ao funcionamento da amígdala dentro do contexto da formação da memória. Na revisão e discussão a seguir, a bibliografia consultada foi distribuída em tópicos com base nos seguintes enfoques: 1) aspectos gerais da memória e regiões cerebrais envolvidas com ênfase na sua relação com estados emocionais; 2) participação da amígdala na memória para eventos emocionalmente relevantes e principais estudos em humanos; e 3) estudos comportamentais e mecanicísticos em modelos animais. 


\section{SCUSSÃO}

Memória: aspectos gerais, modelos animais e relação com medo e ansiedade

O aprendizado e a memória são fenômenos comportamentais relacionados à plasticidade do sistema nervoso central. Sob o aspecto estrutural, o fenômeno do aprendizado é visto como o processo pelo qual os sistemas celulares e moleculares cerebrais transformam uma informação nova em um traço de memória que pode ser armazenado e recuperado ${ }^{4}$.

Um dos maiores avanços conceituais no estudo da cognição foi o da descoberta de que a memória não corresponde a um processo ou entidade unitários. Baseadas nesse conceito, inúmeras classificações dos fenômenos mnemônicos têm sido propostas, e múltiplos sistemas funcionais para a formação de memórias têm sido descritos ${ }^{5-8}$. A memória pode ser classificada de acordo com sua duração ${ }^{5}$, sendo a memória de curto prazo uma forma de memória de capacidade limitada da qual fariam parte elementos de um passado imediato presentes ainda na consciência. Já a memória de longo prazo seria mais estável, sendo constituída por elementos do passado já ausentes da consciência, mas que podem ser evocados por estímulos a eles relacionados. Memórias de longo prazo não seriam formadas instantaneamente, mas consolidadas ao longo do tempo depois do processo de aprendizado ${ }^{9}$. Sugere-se que durante um determinado período do processo de consolidação, a memória permaneceria suscetível a modulações endógenas neuroquímicas por eferências de outras regiões cerebrais para a região na qual está ocorrendo o armazenamento ${ }^{10,11}$.

De acordo com o tipo de informação processada, a memória pode ser classificada em memória declarativa (ou explícita), que compreende o armazenamento de conhecimentos que podem ser conscientemente e intencionalmente recordados (informações sobre locais, pessoas, fatos, etc.), e em memória de procedimento (ou implícita), que se refere às habilidades motoras e perceptivas ${ }^{8}$.

Acredita-se que a memória seja uma função cerebral que envolve diversas regiões, tais como o córtex cerebral e várias estruturas do sistema límbico ${ }^{2,12}$. Entretanto, algumas regiões específicas são mais importantes para determinados tipos de memória. Desde a constatação de que o paciente H. M., após sofrer ablação bilateral do lobo temporal medial, tinha perdido a capacidade de formar novas memórias declarativas ${ }^{13}$, o lobo temporal, mais precisamente a formação hipocampal, foi proposta como sendo responsável pela conversão de memória de curto prazo em memória de longo prazo. Paralelamente, como o paciente H. M. preservava memórias relativas a períodos anteriores à cirurgia, concluiu-se que o hipocampo não seria o responsável pelo armazenamento ou evocação das informações adquiridas, sendo esse papel responsabilidade de outras estruturas. Sugere-se que a memória declarativa de longo prazo seja armazenada no mesmo conjunto de estruturas corticais que participam de seu processamento ${ }^{2,14}$. Contudo, para esse armazenamento, conexões bidirecionais entre essas áreas e aquelas do lobo temporal medial seriam fundamentais ${ }^{15}$. Além disso, parece que diferentes componentes da memória, com diferentes significados, seriam processados paralelamente em diferentes regiões. Por exemplo, estudos utilizando lesões mostraram que a amígdala é responsável pelos processos de alerta e/ou de informação aversiva, participando da memória para eventos com significado emocional (ver adiante).

Embora os mecanismos responsáveis pela formação da memória não sejam completamente conhecidos, acredita-se que a memória esteja associada com alterações plásticas neuronais. Assim, numerosos estudos têm se concentrado na descoberta dos mecanismos celulares e moleculares responsáveis por essas alterações. A ideia de que o traço da memória seria encontrado nas sinapses capazes de sofrer mudanças dependentes de atividade em sua eficiência foi primeiramente apresentada por Konorski ${ }^{16}$ e Hebb ${ }^{17}$. Estes autores propuseram que uma sinapse entre duas células seria fortalecida se as duas células fossem ativadas ao mesmo tempo. Estudos mais recentes têm mostrado que após uma situação de aprendizado ocorre uma reativação neuronal dependente dessa experiência ${ }^{18,19}$. Após tal reverberação neuronal, mudanças plásticas nas sinapses seriam então responsáveis pela consolidação da memória. Nesse contexto, séries de estimulações de alta frequência em vias excitatórias monossinápticas no hipocampo causam um aumento abrupto e duradouro na eficiência da transmissão sináptica ${ }^{20}$. Esse efeito é denominado potencialização de longa duração (long-term potentiation, LTP) e já foi encontrado em todas as vias excitatórias do 
hipocampo e em várias outras regiões do cérebro ${ }^{21-23}$. Esse fenômeno levaria às modificações responsáveis pelo fortalecimento da sinapse e, em última instância, à indução gênica e síntese proteica, processos que embasariam a consolidação e o armazenamento da memória ${ }^{24-28}$.

Independente do completo esclarecimento dos processos neurofisiológicos que envolvem a formação da memória (aquisição, consolidação e evocação), assim como sua extinção (eliminação de uma resposta aprendida), sabe-se que esses processos estão sujeitos à modulação por diversos fatores. 0 contexto emocional da situação é um dos fatores mais relevantes. De fato, inúmeros estudos demonstram que a valência emocional das informações é diretamente proporcional à probabilidade de recordação das mesmas, conforme revisado por LaBar \& Cabeza ${ }^{29}$ e Phelps ${ }^{30}$. As memórias que envolvem estímulos com valência emocional tendem a ser mais duradouras e vívidas, ainda que as memórias declarativas com ou sem conteúdo emocional sejam formadas pelo funcionamento do sistema do lobo temporal medial ${ }^{30,31}$. Um estudo demonstrou que o estado de alerta induzido pelo estímulo a ser lembrado é um forte indicativo do desempenho da memória subsequente ${ }^{32}$.

Sugere-se que essa diferença ocorra devido à modulação da estruturas do lobo temporal medial pela ativação da amígdala durante os processos de codificação e consolidação de uma memória ${ }^{30,33,34}$.

Muito do conhecimento que se tem disponível sobre os mecanismos neurobiológicos da memória é proveniente de estudos com modelos animais, desde invertebrados até primatas não humanos, sendo a grande maioria realizada em roedores. Basicamente dois tipos de aprendizado embasam os modelos animais de memória: aprendizado não associativo e aprendizado associativo ${ }^{35}$. A memória não associativa é uma forma simples de aprendizado que envolve mudança da intensidade da resposta a um estímulo. Em sua maioria, entretanto, os modelos de memória fazem uso do aprendizado associativo, no qual dois estímulos estão associados temporalmente. No caso do condicionamento clássico (ou pavloviano), por exemplo, um estímulo normalmente neutro (condicionado) é pareado com um estímulo efetivo (incondicionado), que pode ser tanto reforçador como aversivo ${ }^{2}$. O condicionamento clássico de respostas de medo é amplamente utilizado como um modelo para investigar tanto os mecanismos neurobiológicos da memória em mamíferos quanto aspectos fisiopatológicos de distúrbios ligados ao medo em humanos ${ }^{36-38}$.

Nos modelos de memória associativa, ocorre participação ativa de mecanismos responsáveis por motivação, recompensa ou medo, uma vez que é necessário o pareamento de uma estimulação aversiva ou apetitiva para que se dê o condicionamento. Nesse sentido, embora a memória muitas vezes seja considerada a mais importante das funções cognitivas, seu funcionamento depende de outras funções cerebrais, tais como vigilância, motivação, atenção. O fato é que, como uma função cognitiva, a memória foi tida como um mecanismo racional diferente e separado daqueles ditos emocionais. A ciência seguiu a tradição platônica de separar a paixão da razão, e as emoções foram colocadas fora da ordem do dia, relegadas ao chamado "fantasma da máquina" - o responsável por todos os fenômenos que a ciência não conseguia investigar empiricamente (um amplo histórico dessa dicotomia pode ser encontrado nos textos de Damásio ${ }^{1}$, LeDoux ${ }^{39}$ e Pinker ${ }^{40}$ ). Entretanto, a partir da segunda metade do século passado, alguns cientistas cognitivos começaram a reconhecer a importância das emoções, e a mente foi definitivamente repatriada ao corpo nos anos 1990, a década do cérebro ${ }^{39}$.

Baseado na premissa de que "as ordens de nível inferior do nosso organismo fazem parte do mesmo circuito que assegura o nível superior da razão", Damásiol sugere que a emoção, como expressão dos mecanismos básicos de regulação biológica, não só orientou o desenvolvimento evolutivo da razão humana, como também é determinante para o adequado funcionamento racional de cada indivíduo. A aproximação ou o retraimento, a excitação ou a quiescência de um organismo em relação a um objeto ou situação decorrem da avaliação emocional desta. Desse modo, a reação emocional é uma resposta que busca criar uma situação mais benéfica para o organismo que garanta sua autopreservação. É evidente que a raiva e o medo salvaram numerosas vidas ao longo da evolução. Portanto, a capacidade de formar memórias emocionais, constituindo emoções secundárias, conferiu ampla vantagem aos organismos. Por outro lado, algumas emoções parecem pouco ou nada adaptativas, como, por exemplo, aquelas envolvidas nas fobias e outros distúrbios de medo, devendo-se ressaltar, nesse sentido, que um processamento inadequado das emoções parece ser um aspecto comum à grande maioria dos distúrbios mentais ${ }^{41,42}$.

Alguns estudos têm proposto que o medo e a memória não seriam apenas fenômenos relacionados, mas que a ansiedade (uma sensação subjetiva relacionada ao medo) seria, na verdade, um passo 
necessário para que ocorresse a formação da memória ${ }^{43}$. Essa possibilidade parece ser apoiada pelo fato de que a administração de ansiolíticos benzodiazepínicos (fármacos que diminuem o medo e a ansiedade) produz amnésia anterógrada ${ }^{44}$, enquanto o estado de alerta produzido por um item a ser lembrado fortalece sua memória ${ }^{32,45}$. Paralelamente, fatores de natureza cognitiva (por exemplo, memórias de experiências anteriores) podem determinar a maneira e a intensidade da expressão das respostas de medo ${ }^{46}$, respostas nas quais muitos modelos animais de memória se baseiam. Nesse sentido, a avaliação específica do efeito de manipulações sobre processos de memória em modelos animais pode se ver seriamente prejudicada se a manipulação em questão apresentar qualquer ação sobre os níveis de ansiedade (medo) no momento dos testes experimentais. Mais especificamente, uma série de estudos de nosso grupo de pesquisa mostrou que alterações bidirecionais no nível basal de ansiedade (o que corresponderia ao medo inato) prejudicam o desempenho de roedores em uma tarefa discriminativa aversiva ${ }^{4-51}$. O modelo utilizado em tais estudos foi o de esquiva discriminativa em labirinto em cruz elevado, que permite a avaliação concomitante do medo inato (por meio da exploração de braços sem paredes) e do aprendizado e da memória de uma tarefa declarativa (discriminação de dois braços fechados, sendo um seguro e o outro associado à estimulação aversiva ${ }^{52}$ ). Dessa forma, tais estudos sugerem a importância de um estado basal emocional ótimo para que ocorra a formação de memórias declarativas relacionadas a situações de perigo potencial.

Deve-se ressaltar que as memórias de eventos aversivos, apesar de desempenharem um importante papel biológico, prestando-se à antecipação de situações potencialmente perigosas, também podem ser responsáveis pelo desencadeamento de respostas patológicas de medo. Foi demonstrado, por exemplo, que a exposição prévia a um estresse psicológico significativo preserva, ou até mesmo melhora, a memória para aspectos emocionais de um determinado evento, enquanto prejudica a memória para aspectos neutros desse mesmo evento ${ }^{53}$. De fato, distúrbios como o transtorno de estresse pós-traumático, o pânico e as fobias têm sido associados a um deficit no processo de extinção de respostas de medo condicionadas ${ }^{36,38,42,54,55}$. Dessa forma, há considerável interesse em esclarecer os mecanismos neurobiológicos do processo de extinção do medo condicionado.

Conforme será discutido a seguir, estudos de neuroimagem ${ }^{34,56,57}$ mostraram que há aumento na atividade da amígdala durante a codificação de eventos emocionais. Além disso, muitos estudos em modelos animais indicam o papel da amígdala na reversão da valência emocional dos estímulos ${ }^{58-62}$.

Amígdala e memória para eventos emocionalmente relevantes

A amígdala ou complexo amigdaloide é um aglomerado heterogêneo de núcleos neurais localizados no lobo temporal ${ }^{63}$. Considerada o centro emocional cerebral ${ }^{37,64-66}$, acredita-se que a amígdala desempenhe um papel chave no planejamento comportamental baseado na integração de informação íntero e exteroceptiva ${ }^{1,39}$. Tem-se atribuído uma grande variedade de funções para o complexo amigdaloide, incluindo memória, atenção, interpretação do significado emocional dos estímulos sensoriais e gênese dos aspectos emocionais dos sonhos ${ }^{67-70}$. A amígdala parece desempenhar um papel crítico no aprendizado sempre que este envolver o condicionamento a um reforço com valor emocional, independente de se tratar de recompensa ou punição, e estudos em humanos, roedores e primatas não humanos sugerem que a atividade neural nessa estrutura é diferencialmente afetada pela valência emocional do estímulo62,71-75.

O primeiro estudo a apontar a participação da amígdala nos processos de consolidação da memória foi o de Weiskrantz ${ }^{66}$, que sugeria que lesões nessa estrutura impediriam a formação de associações estímulo-reforço. Posteriormente, Goddard ${ }^{76}$ mostrou que a estimulação elétrica da amígdala de ratos após o aprendizado promovia um prejuízo grave na retenção da tarefa. Foi sugerido que a estimulação elétrica da amígdala poderia melhorar ou prejudicar a memória, dependendo das condições do treino ${ }^{77}$.

A partir da década passada, inúmeros estudos têm sugerido que a amígdala (especialmente a porção basolateral) seria o local no qual é formada a associação estímulo-resposta que embasa o condicionamento da resposta de medo, sendo permanentemente armazenada (para revisão ver LeDoux ${ }^{36}$ ). Por outro lado, outros estudos levantaram a hipótese de que a amígdala exerceria um 
papel modulador sobre processos de aquisição e consolidação que ocorreriam em outras estruturas ( para revisão ver Cahill \& McGaugh ${ }^{33}$ ). Mais adiante se encontra uma discussão mais detalhada de tais teorias, bem como dos modelos animais utilizados para embasá-las.

Quando falamos sobre o papel da amígdala na memória emocional, dois aspectos podem ser abordados. O primeiro seria o da participação dessa estrutura na formação de respostas condicionadas a determinados estímulos, inicialmente neutros, que seriam temporalmente associados a estímulos eliciadores primários de tais respostas. Nesse caso, assume-se que a resposta pode ser emitida sem a recordação consciente da situação de aquisição, ou seja, teríamos uma memória implícita. Nesse sentido, evidências mostram que para que a amígdala seja ativada não é necessária a percepção consciente do estímulo ${ }^{78-80}$. O segundo aspecto a ser considerado é o do papel da amígdala na formação de memórias declarativas, ou seja, naquelas memórias que requerem o recordar consciente para serem evocadas. Nesse caso, preconiza-se que a amígdala, ativada pelo conteúdo emocional dos eventos, influenciaria a codificação e posterior evocação das memórias.

Enquanto a participação da amígdala em respostas emocionais condicionadas a eventos aversivos tem sido mostrada inequivocamente tanto em modelos animais (ver adiante) quanto em humanos ${ }^{81-84}$, os estudos que procuram investigar a participação da amígdala na memória declarativa em humanos mostram-se controversos. Scoville \& Milner ${ }^{13}$, examinando 10 pacientes com diferentes extensões de remoção bilateral do lobo temporal medial, concluíram que a remoção da amígdala não parece causar deficit de memória, uma vez que o deficit em questão apareceu apenas em pacientes nos quais alguma porção do hipocampo tinha sido removida. Mais recentemente, um estudo mostrou que a amígdala é importante para o condicionamento clássico de respostas autonômicas, mas não está envolvida com a memória declarativa ${ }^{85}$.

Entretanto, alguns estudos em raros pacientes com lesão seletiva de amígdala ${ }^{86}$ e estudos com imageamento cerebral corroboram a hipótese de que a atividade na amígdala mediaria a memória para eventos emocionais. Em um estudo de imageamento cerebral por PET scan, a atividade cerebral na amígdala durante um filme de conteúdo emocional mostrou alta correlação com a subsequente recordação do mesmo, o que não ocorreu no caso de filmes neutros ${ }^{56}$. LaBar et al. ${ }^{87}$ demonstraram em um estudo de ressonância magnética funcional que a amígdala participa tanto da aquisição quando do processo de extinção de respostas condicionadas de medo. Mostrou-se ainda que em tais pacientes com lesão de amígdala a memória para eventos não emocionais se encontra inalterada e que reações emocionais que independam de recordação podem permanecer normais, sugerindo que a amígdala pode não ser crítica para a reação emocional per se, mas por processos que traduzem a reação emocional em informação que pode ser evocada em longo prazo $33,56,86,88$. Foi demonstrado, ainda, que a evocação correta de eventos apresentados em contextos emocionais está associada à atividade na amígdala ${ }^{89,90}$. Já o estudo de Erk et al. ${ }^{91}$ sugere que essa associação é específica para situações com conteúdo negativo.

Reforçando a hipótese de que a amígdala exerceria seu papel na memória emocional por modular a atividade de outras estruturas, um estudo mostrou que a ação da amígdala depende da integridade de suas interconexões com o hipocampo ${ }^{15,92}$. Além disso, a partir dos resultados obtidos por imageamento cerebral por PET scan, Kilpatrick \& Cahill ${ }^{93}$ demonstraram que há um aumento significativo da influência da amígdala no giro para-hipocampal e no córtex pré-frontal durante a apresentação de filmes de conteúdo emocional relevante (em comparação a filmes neutros).

Por outro lado, outros estudos sugerem que a amígdala humana (especialmente a esquerda) estaria envolvida na responsividade emocional a vários estímulos aversivos ${ }^{94-97}$. Um outro estudo realizado com PET scan relatou que assistir a um filme com conteúdo emocional promove a ativação da amígdala (presumivelmente com consequente formação de memória), mas que a recordação do mesmo não o faz ${ }^{98}$. Contudo, Dolan et al. ${ }^{89}$ encontraram ativação da amígdala na recordação de objetos ou cenas emocionais.

Shibuya-Tayoshi et al. ${ }^{99}$ descreveram uma demência pré-senil na qual distúrbios emocionais eram predominantes a alterações de memória. O quadro mostrou-se decorrente de uma rara degeneração idiopática localizada na amígdala. Outro estudo demonstrou ainda que um paciente com lesão bilateral de amígdala (e com grave deficit na percepção de emoções) mostrou um padrão normal de potencialização da memória para eventos emocionais em relação à memória para material 
neutro ${ }^{100}$. É interessante apontar que idosos saudáveis mantêm o mesmo padrão de memória emocional, ainda que ocorra um declínio da memória explícita ${ }^{101}$.

Por fim, embora os estudos acerca da participação da amígdala na memória em humanos sejam numerosos e tragam avanços para o esclarecimento dessa questão, deve-se ressaltar como limitações desses estudos a escassez de indivíduos com lesão bilateral restrita às amígdalas e o fato de a correlação encontrada entre a atividade neural identificada por imageamento cerebral e determinados eventos não necessariamente representar uma relação causal.

Participação da amígdala na memória para eventos aversivos: modelos animais

Os circuitos complexos entre os diversos núcleos amigdaloides permitem o processamento em paralelo da representação dos estímulos, possibilitando a modulação de tal representação por diversos sistemas funcionais ${ }^{102}$. No que diz respeito ao processamento de memória aversiva ou resposta condicionada de medo, diversas evidências indicam a participação do complexo basolateral (que integraria os estímulos sensoriais e é formado pelos núcleos lateral, basal e basal acessório) e do núcleo central (que, recebendo entradas de outras regiões da amígdala, inclusive do complexo basolateral, seria responsável pela emissão da resposta) ${ }^{103-106}$. Especificamente em relação à resposta de medo condicionada por um protocolo de condicionamento pavloviano, acredita-se que o núcleo lateral é o sítio de convergência de vias neurais que trazem informações sobre os estímulos condicionado e incondicionado ${ }^{39}$. A expressão emocional dessa associação aprendida seria mediada por conexões entre os núcleos lateral e central; por meio de projeções hipotalâmicas e para o tronco cerebral, o segundo coordenaria as respostas comportamentais, autonômicas e endócrinas envolvidas na reação emocional ${ }^{107}$. Por outro lado, Killcross et al. ${ }^{108}$ sugeriram que o núcleo central e o complexo basolateral, ao invés de interagirem para o aprendizado e execução de uma resposta de medo condicionada, mediariam tipos diferentes de respostas de medo. Esses autores mostraram, em ratos, que a lesão do núcleo central promove uma inibição da resposta condicionada pavloviana, enquanto a lesão do complexo basolateral impede o aprendizado associativo da esquiva a um estímulo aversivo. Esse último tipo de aprendizado envolveria, ao contrário do condicionamento pavloviano, um componente de memória declarativa.

Estudos em modelos animais (principalmente em roedores) deram origem a duas hipóteses sobre a participação da amígdala na memória aversiva: 1) essa estrutura seria o local dos processos plásticos envolvidos na aquisição e consolidação dessas memórias ${ }^{36,55}$ e 2) essas estrutura exerceria um papel de modulação sobre os processos de aquisição e consolidação que estariam ocorrendo em outras estruturas $33,109,110$.

Os estudos que levaram à elaboração da primeira hipótese são baseados no modelo de resposta de medo condicionada a um som ou contexto ${ }^{36}$. Nesse paradigma de condicionamento pavloviano, ratos são submetidos a um pareamento de um estímulo condicionado (o som ou o contexto) com um choque elétrico aplicado às patas (estímulo incondicionado). Após certo número de pareamentos, passam a apresentar a resposta que normalmente apresentariam ao choque apenas com a apresentação do som ou do contexto. Preconiza-se que nesse tipo de condicionamento o estímulo condicionado torna-se associado a um estado emocional interno de medo eliciado pelo estímulo incondicionado. A resposta, denominada freezing (congelamento), é caracterizada por ausência total de movimentos com exceção dos movimentos respiratórios, sendo uma reação de medo característica da espécie ${ }^{111,112}$. Tais estudos mostraram que lesões ou inativações temporárias da amígdala, especialmente no complexo basolateral, levam tanto a um deficit de desempenho na tarefa de medo condicionado como a um prejuízo na evocação e na extinção dessa tarefa (processo no qual ocorreria mudança do significado emocional do estímulo) $36,39,58,113-119$. Ainda corroborando essa hipótese, estudos mostram a ocorrência do fenômeno de LTP nas vias tálamo-amigdalar ${ }^{120,121}$ e hipocampo-amigdalar ${ }^{122}$, envolvidas com o processamento do medo condicionado ao som e ao contexto, respectivamente ${ }^{36}$. Schroeder $\&$ Shinnick-Gallagher ${ }^{123}$, mostrando a presença de modificações na eficiência sináptica da via corticoamigdalar 10 dias após o condicionamento da resposta de medo, sugeriram que essa via estaria envolvida com o armazenamento a longo prazo das memórias aversivas. Yeh et al. ${ }^{124}$ demonstram um aumento da expressão sináptica de receptores glutamatérgicos GluR1 na amígdala de ratos submetidos a uma situação de resposta 
condicionada de medo. Além disso, a expressão de alguns genes no núcleo lateral da amígdala parece estar relacionada especificamente com a resposta condicionada de medo ${ }^{125}$.

Maren \& Quirk ${ }^{55}$ revisaram estudos eletrofisiológicos que indicam que o complexo basolateral da amígdala, mais especificamente o núcleo lateral, seria o local dos mecanismos plásticos que codificariam a memória aversiva durante a aquisição e a extinção do condicionamento pavloviano. Nessa revisão, um destaque foi dado a resultados de alguns estudos de registro de atividade neuronal unitária na amígdala 59,117,126-129. Entretanto, dados desse tipo de registro envolvendo outras situações de aprendizado são mais raros. Coerentemente com a ausência de uma participação crítica da amígdala na formação da memória aversiva, o estudo de Naylor et al. ${ }^{130}$ mostrou que a inibição da atividade espontânea do núcleo central da amígdala (demonstrada pelo registro de atividade neuronal unitária) não promoveu deficits de retenção no modelo da esquiva passiva. Entretanto, outros estudos de registro de atividade neuronal propõem uma correlação entre atividade neuronal na amígdala e outras estruturas, como o córtex orbitofrontal e o córtex piriforme, sugerindo um funcionamento conjunto em processos de aprendizado ${ }^{131,132}$.

Apesar da grande quantidade de estudos baseados nesse paradigma, o uso do modelo de medo condicionado para avaliação da participação da amígdala na memória aversiva tem sido questionado ${ }^{33,110,133-138}$ em vista de alguns fatores. Primeiramente, lesões ou outros procedimentos que impedem o funcionamento da amígdala promovem aumento da atividade motora ${ }^{111,139,140} \mathrm{e}$ uma diminuição do medo não aprendido ou inato $137,142-145$ (mas ver o estudo de Antoniadis \& McDonald ${ }^{146}$ ). Nesse sentido, enquanto um aumento da atividade motora dificultaria a ocorrência de uma resposta dependente de imobilidade, uma diminuição do desempenho na tarefa poderia significar uma redução do "significado aversivo" do estímulo ao invés de um deficit nos processos mnemônicos. Além disso, a amígdala parece ser importante para a expressão da resposta de freezing ${ }^{111,137}$, e o estudo dos efeitos de uma manipulação sobre o aprendizado e a memória requer que tal manipulação não afete o comportamento utilizado para avaliá-los. Finalmente, as informações obtidas com base somente em estudos de condicionamento pavloviano parecem ser insuficientes porque as memórias emocionalmente influenciadas geralmente envolvem conhecimento declarativo e não estão restritas às evocações de respostas emocionais aprendidas ${ }^{33}$. Nesse sentido, existe uma clara dissociação entre o condicionamento pavloviano e a memória declarativa ${ }^{147,148}$. Dessa forma, questiona-se se os mecanismos neurobiológicos elucidados para a resposta de medo condicionada poderiam ser extrapolados para situações de processamento de memória declarativa com conteúdo emocional relevante. Em um estudo recente, Paz et al. ${ }^{75}$ mostraram que a ativação da amígdala basolateral aumenta o processamento sensorial durante eventos emocionalmente relevantes por meio da facilitação da transmissão neural entre os córtices perirrinal e entorrinal. Além disso, essa facilitação mostrou alta correlação com o aprendizado, corroborando a hipótese de que a amígdala exerceria um papel de modulação sobre os processos de aquisição e consolidação (que estariam ocorrendo em outras estruturas) para informações com conteúdo emocional relevante.

A maioria dos estudos que corroboram a hipótese de que amígdala atua modulando a atividade em outras estruturas foi realizada no modelo de esquiva passiva em roedores. Tal modelo, a princípio, difere da resposta emocional condicionada descrita anteriormente. Nesse paradigma, ratos são colocados em um local diferente daquele no qual receberão o estímulo aversivo (choque elétrico nas patas), recebendo tal estimulação apenas ao se transferirem voluntariamente para o compartimento "aversivo". A memória é avaliada pelo aumento da latência para transferência para o compartimento no qual o animal recebeu o choque em uma posterior reexposição. Nesse tipo de procedimento, o desempenho do animal permite a inferência de que ocorre uma memória de natureza declarativa para a situação específica ${ }^{33}$. Tais estudos apontaram para uma participação moduladora, mas não crítica, da amígdala na memória aversiva, mostrando que: 1) a inativação da amígdala promove atenuação, mas não bloqueio da retenção da tarefa; 2) essa atenuação só ocorre se a manipulação para impedir o funcionamento da amígdala for realizada antes ou até um tempo limitado depois do treino; 3) a inativação da amígdala impede a modulação da memória exercida por hormônios do estresse e catecolaminas; 4) efeitos de manipulações em outras estruturas como o córtex insular e o hipocampo sobre a memória sofrem modulação da atividade na amígdala e 5) manipulações da atividade da amígdala basolateral modulam a expressão de proteínas relacionadas à plasticidade no hipocampo 33,110,133,135-137,149-155. 
Embora a utilização da esquiva passiva em roedores para o estudo do papel da amígdala na memória aversiva contemple alguns pontos críticos do uso do medo condicionado para esse fim (a avaliação do desempenho por uma resposta diferente da resposta de freezing e acréscimo do caráter declarativo da tarefa), alguns fatores ainda podem interferir com a interpretação dos resultados encontrados nesse modelo. Assim, o aumento da atividade motora promovido pela inativação da amígdala ${ }^{111,139,140}$ poderia promover per se uma diminuição na latência de transferência do animal. Já uma diminuição do medo não aprendido ou inato ${ }^{137,141-145}$ poderia diminuir a relevância do estímulo incondicionado e prejudicar o processo de condicionamento indiretamente.

De especial relevância dentro desse contexto são resultados recentes de nosso grupo que mostram, em uma sessão de teste típica da tarefa de esquiva passiva, que ratos que haviam sido expostos diretamente ao compartimento do choque no dia do treino (da mesma forma que em uma tarefa de medo condicionado) apresentam desempenho semelhante ao dos animais que haviam recebido um treino convencional de esquiva passiva, recebendo o estímulo apenas ao transferir-se voluntariamente para o compartimento do choque (Silva et al., dados não publicados).

Esse resultado leva ao questionamento quanto a se os dois modelos cujos resultados embasam diferentes teorias a respeito da participação da amígdala na memória aversiva seriam mesmo tarefas diferentes.

Os estudos de Vazdarjanova \& McGaugh ${ }^{137}$ e LaLumiere et al. ${ }^{156}$ se propõem a investigar o envolvimento do complexo basolateral da amígdala na memória aversiva em uma adaptação do modelo de medo condicionado ao contexto na qual são realizadas outras medidas além da resposta de freezing. O modelo é realizado em um labirinto em $Y$, com três braços fechados diferentes, realizando-se o pareamento do choque ao contexto confinando os animais em um dos braços. No teste, os animais podem explorar livremente o labirinto, e o desempenho é avaliado pelo tempo de exploração do braço do choque. Essa exploração foi diminuída, mas não bloqueada, pela inativação da amígdala, corroborando a teoria do papel modulador dessa estrutura na memória aversiva. Esse protocolo, além de permitir a avaliação da memória por uma resposta diferente da de freezing, também permitiu a dissociação dos efeitos da lesão de amígdala na atividade motora, uma vez que esta pareceu não interferir com o decréscimo da exploração do braço do choque.

Os modelos da esquiva passiva e principalmente do medo condicionado modificado (descrito acima) parecem fornecer melhores ferramentas de investigação do papel da amígdala na memória.

Entretanto, deve-se ressaltar que eles não diferem do modelo do medo condicionado em um ponto importante: a natureza do estímulo aversivo. Nos três modelos, um choque elétrico é aplicado às patas do rato com o objetivo de conseguir-se o condicionamento. Além do explícito aspecto ético, a utilização do choque parece ser desfavorecida para avaliação de memória aversiva tendo em vista o fato de que a hipoalgesia é um componente da resposta de medo nessa espécie ${ }^{146}$. Em outras palavras, uma diminuição da sensação dolorosa poderia estar relacionada com possíveis modificações na resposta.

Finalmente, conforme já comentado, um ponto relevante para a investigação dos efeitos da amígdala na memória é a diminuição da resposta de medo espontânea decorrente da inativação da amígdala (ver acima). Mais importante, estudos mostram que a amígdala é parte integrante dos circuitos neurais que modulam as respostas inatas de medo ${ }^{111,112}$. Nesse sentido, a sinalização neuronal mediada pela expressão gênica na amígdala parece estar relacionada tanto a respostas condicionadas de medo quanto a respostas características de medo inato ${ }^{157}$. Ainda, questiona-se se o papel dos circuitos da amígdala em organizar as respostas inatas de medo seria semelhante ao papel dessa estrutura no processamento de respostas de medo aprendidas ${ }^{112,146,158}$. Por outro lado, diversos estudos demonstraram que o núcleo central da amígdala tem um papel chave para organizar as respostas de medo condicionadas, enquanto outros sugerem que esse núcleo não está envolvido na organização das respostas comportamentais em situações que evocam medo inato ${ }^{159}$.

\section{CONCLUSÃO}

Apesar de os trabalhos aqui revisados fornecerem fortes evidências de que a amígdala modula as estruturas relacionadas com a consolidação da memória, concluímos que o mecanismo que embasa 
tal relação necessita de mais estudos para ser completamente esclarecido. Estudos em humanos sugerem que a ativação da amígdala, embora fundamental nas repostas emocionais, não exerce um papel determinante para a formação da memória, o que não significa que não possa influenciar memórias com conteúdo emocional. Apesar dos inúmeros importantes achados de estudos com modelos animais, parece que em nenhum deles os protocolos permitem separar completamente os efeitos de manipulações na memória aversiva e na resposta inata de medo. "Aprender emoções" confere vantagens evolutivas, e o estudo dos mecanismos desse aprendizado passa necessariamente pela sugestão da amígdala como estrutura chave. Esse esclarecimento definitivo parece depender da possibilidade de se estudar separadamente emoções inatas e aprendidas. Tal separação conceitual permitiria o estudo mais direto das bases biológicas de ambos os fenômenos. Por outro lado, questiona-se se tal tentativa de considerar processos cognitivos e emoções inatas como entidades fisiologicamente isoladas não nos remeteria a uma antiga dicotomia.

\section{REFERÊNCIAS}

1. Damásio A. Descartes Error: Emotion, Reason, and the Human Brain. New York: Putnam; 1994.

2. Squire LR, Kandel ER. Memory: from mind to molecules. New York: W.H. Freeman and Company; 2000.

3. Broglio C, Gomez A, Duran E, Ocaña FM, Jiménez-Moya F, Rodríguez F, et al. Hallmarks of a common forebrain vertebrate plan: specialized pallial areas for spatial, temporal and emotional memory in actinopterygian fish. Brain Res Bull. 2005;66(4-6):277-81.

4. Schmidt R. Cell-adhesion molecules in memory formation. Behav Brain Res. 1995;66:65-72.

5. Baddeley AD, Warrington EK. Amnesia and the distinction between long and short-term memory. J Verbal Learn Verbal Behav. 1970;9:176-89.

6. Oliveira MG, Bueno OF. Neuropsicologia da memória humana. Psicologia USP. 1993;4:117-38.

7. Tulving E. Organization of memory: quo vadis? In: Gazzaniga MS, ed. The Cognitive Neuroscience. Cambridge: MIT Press; 1995. p. 839-847.

8. Squire LR. Memory systems of the brain: A brief history and current perspective. Neurobiol Learn Mem. 2004; 82:171-7.

9. McGaugh JL. Time-dependent processes in memory storage. Science. 1966;153(742): 1351-8.

10. Alberini CM, Milekic MH, Tronel S. Mechanisms of memory stabilization and de-stabilization. Cell Mol Life Sci. 2006;63(9):999-1008.

11. Izquierdo I, Bevilaqua LR, Rossato JI, Bonini JS, Medina JH, Cammarota M. Different molecular cascades in different sites of the brain control memory consolidation.Trends Neurosci.

2006; 29(9): 496-505.

12. Squire LR. Memory and brain. Oxford: Oxford University Press; 1987.

13. Scoville WB, Milner B. Loss of recent memory after bilateral hippocampus lesions. J Neurol Neurosurg Psychiatr. 1957; 20:11-21.

14. Jerusalinsky D, Kornisiuk E, Izquierdo I. Cholinergic neurotransmission and synaptic plasticity concerning memory processing. Neurochem Res. 1997;22:507-15.

15. Eichenbaum H. A cortical-hippocampal system for declarative memory. Nature Rev Neurosci. 2000; 1:41-50.

16. Konorski J. Conditioned reflexes and neuron organization, Cambridge: Cambridge University Press; 1948. 
17. Hebb DO. The Organization of Behavior. New York: John Wiley Inc; 1949.

18. Pavlides C, Winson J. Influences of hippocampal place cell firing in the awake state on the activity of these cells during subsequent sleep episodes. J Neurosci. 1989;9(8):2907-18.

19. Ribeiro S, Gervasoni D, Soares ES, Zhou Y, Lin SC, Pantoja J, et al. Long-lasting noveltyinduced neuronal reverberation during slow-wave sleep in multiple forebrain areas. PLoS Biol. 2004;2(1): E24.

20. Bliss TV, Lomo T. Long-lasting potentiation of synaptic transmission in the dentate area of the anaesthetized rabbit following stimulation of the perfront path. J Physiol. 1973;232:331-56.

21. Doyere $\mathrm{V}$, LaRoche S. Linear relationship between the maintenance of hippocampal long-term potentiation and retention of associative memory. Hippocampus. 1992;2:39-48.

22. Rioult-Pedotti MS, Friedman D, Donoghue JP. Learning-induced LTP in neocortex. Science. 2000;290(5491):533-6.

23. Yaniv D, Richter-Levin G. LTP in the rat basal amygdala induced by perirhinal cortex stimulation in vivo. Neuroreport. 2000;11(3):525-30.

24. Jodar L, Kaneto H. Synaptic plasticity: stairway to memory. Jpn J Pharmacol 1995;68:359-87.

25. Huang YY, Nguyen PV, Abel T, Kandel ER. Long-lasting forms of synaptic potentiation in the mammalian hippocampus. Learn Mem. 1996;3(2-3):74-85.

26. Kandel ER. Genes, synapses, and long-term memory. J Cell Physiol. 1997;173(2):124-5.

27. Hölscher C. Nitric oxide is required for expression of LTP that is induced by stimulation phaselocked with theta rhythm. Eur J Neurosci. 1999;11:335-43.

28. Chen C, Magee JC, Marcheselli V, Hardy M, Bazan NG. Attenuated LTP in hippocampal dentate gyrus neurons of mice deficient in PAF receptor. J Neurophysiol. 2001;85:384-90.

29. LaBar KS, Cabeza R. Cognitive neuroscience of emotional memory. Nat Rev Neurosci. 2006; 7(1):54-64.

30. Phelps EA. Emotion and cognition: insights from studies of the human amygdala. Ann Rev Psychol. 2006; 57:27-53.

31. Christianson S. The handbook of emotion and memory: research and theory. London: Lawrence Erlbaum Associates; 1992.

32. Buchannan TW, Etzel J A, Adolphs R, Tranel D. The influence of autonomic arousal and semantic relatedness ons memory for emotional words. Int J Psychophysiol. 2006;61:26-33.

33. Cahill L, McGaugh JL. Mechanisms of emotional arousal and lasting declarative memory. Trends Neurosci. 1998;21(7):294-9.

34. Hamann SB. Cognitive and neural mechanisms of emotional memory. Trends Cog Sci. 2001;5(9):394-400.

35. Levitan IB, Kaczmarek LK. Learning and memory. In: Levitan IB, Kaczmarek LK, eds. The Neuron: Cell and Molecular Biology. Oxford: Oxford University Press; 1991. p. 395-423.

36. LeDoux JE. Emotion circuits in the brain. Ann Rev Neurosci. 2000;23:155-84.

37. Kim JJ, Jung MW. Neural circuits and mechanisms involved in Pavlovian fear conditioning: a critical review. Neurosci Biobehav Rev. 2006;30(2):188-202.

38. Rauch SL, Shin LM, Phelps EA. Neurocircuitry models of posttraumatic stress disorder and extinction: human neuroimaging research - past, present, and future. Biol Psychiatry. 
2006;60(4):376-82.

39. LeDoux JE. The emotional brain. New York: Simon and Schuster; 1996.

40. Pinker S. The blank slate: the modern denial of human nature. New York: Penguin Books; 2002.

41. Dolan RJ. Emotion, cognition, and behavior. Science. 2002;298(5596): 1191-4.

42. Maren S. Building and burying fear memories in the brain. Neuroscientist. 2005;11(1):89-99.

43. Mathews A. Why worry? The cognitive function of anxiety. Behav Res Ther. 1990;28:455-568.

44. Thiebot MH. Some evidence for amnestic-like effects of benzodiazepines in animals. Neurosci Behav Rev. 1985; 9:95-100.

45. Anderson AK, Wais PE, Gabrieli JDE. Emotion enhances remembrance of neutral events past. PNAS. 2006; 103(5): 1599-604.

46. Blanchard RJ, Blanchard DC. An ethoexperimental approach to the study of fear. Psychol Rev.1987;37:302-16.

47. Silva RH, Frussa-Filho R. The plus-maze discriminative avoidance task: a new model to study memory-anxiety interactions. Effects of chlordiazepoxide and caffeine. J Neurosci Methods. 2000;102(2): 117-25.

48. Silva RH, Frussa-Filho R. Naltrexone potentiates both amnestic and anxiolytic effects of chlordiazepoxide in mice. Life Sci. 2002; 72(6):721-30.

49. Silva RH, Kameda SR, Carvalho RC, Rigo GS, Costa KL, Taricano ID, et al. Effects of amphetamine on the plus-maze discriminative avoidance task in mice. Psychopharmacology (Berl). 2002; 160(1): 9-18.

50. Silva RH, Chehin AB, Kameda SR, Takatsu-Coleman AL, Abilio VC, Tufik S, et al. Effects of preor post-training paradoxical sleep deprivation on two animal models of learning and memory in mice. Neurobiol Learn Mem. 2004;82(2):90-8.

51. Calzavara MB, Lopez GB, Abilio VC, Silva RH, Frussa-Filho R. Role of anxiety levels in memory performance of spontaneously hypertensive rats. Behav Pharmacol. 2004;15(8):545-53.

52. Silva RH, Bellot RG, Vital MA, Frussa-Filho R. Effects of long-term ganglioside GM1 administration on a new discriminative avoidance test in normal adult mice. Psychopharmacology. $1997 ; 129(4): 322-8$.

53. Payne JD, Jackson ED, Ryan L, Hoscheidt S, Jacobs JW, Nadel L. The impact of stress on neutral and emotional aspects of episodic memory. Memory. 2006; 14(1):1-16.

54. Pitman RK, Orr SP, Shalev AY, Metzger LJ, Mellman TA. Psychophysiological alterations in post-traumatic stress disorder. Semin Clin Neuropsychiatry. 1999;4(4):234-41.

55. Maren S, Quirk GJ . Neuronal signalling of fear memory. Nat Rev Neurosci. 2004;5(11):844-52.

56. Cahill L, Haier RJ, Fallon J, Alkire MT, Tang C, Keator D, et al. Amygdala activity at encoding correlated with long-term, free recall of emotional information. Proc Natl Acad Sci USA. 1996; 93(15): 8016-21.

57. Adolphs R. Neural systems for recognizing emotion. Curr Opin Neurobiol. 2002; 12:169-77.

58. Falls WA, Miserendino MJ, Davis M. Extinction of fear-potentiated startle: blockade by infusion of an NMDA antagonist into the amygdala. J Neurosci. 1992;12(3):854-63.

59. Quirk GJ, Repa C, LeDoux JE. Fear conditioning enhances short-latency auditory responses of lateral amygdala neurons: parallel recordings in the freely behaving rat. Neuron. 
1995; 15(5): 1029-39.

60. Lu KT, Walker DL, Davis M. Mitogen-activated protein kinase cascade in the basolateral nucleus of amygdala is involved in extinction of fear-potentiated startle. J Neurosci. 2001;21(16): RC162.

61. Repa JC, Muller J, Apergis J, Desrochers TM, Zhou Y, LeDoux JE. Two different lateral amygdala cell populations contribute to the initiation and storage of memory. Nat Neurosci.

$2001 ; 4(7): 724-31$.

62. Paton JJ, Belova MA, Morrison SE, Salzman CD. The primate amygdala represents the positive and negative value of visual stimuli during learning. Nature. 2006;439(7078):865-70.

63. Johnston JB. Further contributions to the study of the evolution of the forebrain. J Comp Neurol. 1923; 35:337-481.

64. Kluver H, Bucy PC. "Psychic blindness" and other symptoms following bilateral temporal lobectomy in rhesus monkeys. Am J Phsysiol. 1937; 119:352-3.

65. McLean PD, Delgado JM. Electrical and chemical stimulation of frontotemporal portion of limbic system in the waking animal. Electroencephalogr. Clin Neuropsychol. 1953; Suppl 5:91-100.

66. Weiskrantz L. Behavioral changes associated with ablation of the amygdaloid complex in monkeys. J Comp Pshysiol Psychol. 1956;49:381-91.

67. Aggleton JP. The contribution of the amygdala to normal and abnormal emotional states. Trends Neurosci. 1993; 16(8):328-33.

68. Adolphs R, Tranel D, Damasio H, Damasio A. Impaired recognition of emotion in facial expressions following bilateraldamage to the human amygdala. Nature. 1994;372(6507):669-72.

69. Maquet P, Peters J, Aerts J, Delgiore G, Degueldre C, Luxen A, et al. Functional neuroanatomy of human rapid-eye-movement sleep and dreaming. Nature. 1996;383(6596): 163-6.

70. Scott SK, Young AW, Calder AJ, Hellawell DJ, Aggleton JP, J ohnson M. Impaired auditory recognition of fear and anger following bilateral amygdala lesions. Nature. 1997;385(6613):254-7.

71. Willick ML, Kokkinidis L. Cocaine enhances the expression of fear-potentiated startle: evaluation of state-dependent extinction and the shock-sensitization of acoustic startle. Behav Neurosci. 1995; 109(5): 929-38.

72. Whalen PJ, Rauch SL, Etcoff NL, Mclnerney SC, Lee MB, Jenike MA. Masked presentations of emotional facial expressions modulate amygdala activity without explicit knowledge. J Neurosci. $1998 ; 18(1): 411-8$.

73. Parkinson JA, Robbins TW, Everitt BJ. Dissociable roles of the central and basolateral amygdala in appetitive emotional learning. Eur J Neurosci. 2000; 12(1):405-13.

74. O'Doherty JP, Deichmann R, Critchley HD, Dolan RJ. Neural responses during anticipation of a primary taste reward. Neuron. 2002;33(5):815-26.

75. Paz R, Pelletier JG, Bauer EP, Pare D. Emotional enhancement of memory via amygdala-driven facilitation of rhinal interactions. Nat Neurosci. 2006;9(10):1321-9.

76. Goddard GV. Amygdaloid stimulation and learning in the rat. J Comp Physiol Psychol. 1964;58:23-30.

77. Gold PE, Hankins L, Edwards RM, Chester J, McGaugh J L. Memory interference and facilitation with posttrial amygdala stimulation: effect on memory varies with footshock level. Brain Res. 1975;86(3):509-13.

78. Morris JS, Ohman A, Dolan RJ . Conscious and unconscious emotional learning in the human amygdala. Nature. 1998;393(6684):467-70. 
79. Morris JS, DeGelder B, Weiskrantz L, Dolan RJ. Differential extrageniculostriate and amygdala responses to presentation of emotional faces in a cortically blind field. Brain. 2001; 124(Pt 6): 1241-52.

80. de Gelder B, Morris JS, Dolan RJ. Unconscious fear influences emotional awareness of faces and voices. Proc Natl Acad Sci USA. 2005; 102(51): 18682-7.

81. LaBar KS, LeDoux JE. Partial disruption of fear conditioning in rats with unilateral amygdala damage: correspondence with unilateral temporal lobectomy in humans. Behav Neurosci. 1996;110(5): 991-7.

82. Cheng DT, Knight DC, Smith CN, Stein EA, Helmstetter FJ. Functional MRI of human amygdala activity during Pavlovian fear conditioning: stimulus processing versus response expression. Behav Neurosci. 2003; 117(1):3-10.

83. Knight DC, Smith CN, Cheng DT, Stein EA, Helmstetter FJ. Amygdala and hippocampal activity during acquisition and extinction of human fear conditioning. Cogn Affect Behav Neurosci. 2004;4(3):317-25.

84. Morris JS, Dolan RJ. Dissociable amygdala and orbitofrontal responses during reversal fear conditioning. Neuroimage. 2004;22(1):372-80.

85. Bechara A, Tranel D, Damasio H, Adolphs R, Rockland C, Damasio AR. Double dissociation of conditioning and declarative knowledge relative to the amygdala and hippocampus in humans. Science. 1995;269(5227): 1115-8.

86. Adolphs R, Cahill L, Schul R, Babinsky R. Impaired declarative memory for emotional material following bilateral amygdala damage in humans. Learn Mem. 1997;4(3):291-300.

87. LaBar KS, Gatenby JC, Gore JC, LeDoux JE, Phelps EA. Human amygdala activation during conditioned fear acquisition and extinction: a mixed-trial fMRI study. Neuron. 1998;20(5):937-45.

88. Hamann SB, Cahill L, Squire LR. Emotional perception and memory in amnesia. Neuropsychology. 1997;11(1): 104-13.

89. Dolan RJ, Lane R, Chua P, Flatcher P. Dissociable temporal lobe activations during emotional episodic memory retrieval. Neuroimage. 2000;11:203-9.

90. Smith AP, Henson RN, Rugg MD, Dolan RJ. Modulation of retrieval processing reflects accuracy of emotional source memory. Learn Mem. 2005; 12(5):472-9.

91. Erk S, Kiefer M, Grothe J, Wunderlich AP, Spitzer M, Walter H. Emotional context modulates subsequent memory effect. Neuroimage. 2003;18(2):439-47.

92. Richardson MP, Strange BA, Dolan RJ. Encoding of emotional memories depends on amygdala and hippocampus and their interactions. Nat Neurosci. 2004;7(3):278-85.

93. Kilpatrick L, Cahill L. Amygdala modulation of parahippocampal and frontal regions during emotionally influenced memory storage. Neuroimage. 2003;20(4):2091-9.

94. Breiter HC, Etcoff NL, Whalen PJ, Kennedy WA, Rauch SL, Buckner RL, et al. Response and habituation of the human amygdala during visual processing of facial expression. Neuron.

$1996 ; 17(5): 875-87$.

95. Irwin W, Davidson RJ, Lowe MJ, Mock BJ, Sorenson JA, Turski PA. Human amygdala activation detected with echo-planar functional magnetic resonance imaging. Neuroreport.

1996; 7(11): 1765-9.

96. Ketter TA, Andreason PJ, George MS, Lee C, Gill DS, Parekh PI, et al. Anterior paralimbic mediation of procaine-induced emotional and psychosensory experiences. Arch Gen Psychiatry. 1996;53(1):59-69. 
97. Zald DH, Pardo JV. Emotion, olfaction, and the human amygdala: amygdala activation during aversive olfactory stimulation. Proc Natl Acad Sci USA. 1997;94(8):4119-24.

98. Reiman EM, Lane RD, Ahern GL, Schwartz GE, Davidson RJ, Friston KJ, et al. Neuroanatomical correlates of externally and internally generated human emotion. Am J Psychiatry.

$1997 ; 154(7): 918-25$.

99. Shibuya-Tayoshi S, Tsuchiya K, Seki Y, Arai T, Kasahara T. Presenile dementia mimicking Picks disease: an autopsy case of localized amygdala degeneration with character change and emotional disorder. Neuropathology. 2005;25(3):235-40.

100. Papps BP, Calder AJ, Young AW, O'Carroll RE. Dissociation of affective modulation of recollective and perceptual experience following amygdala damage. J Neurol Neurosurg Psychiatry. $2003 ; 74(2): 253-4$.

101. Denburg NL, Buchanan TW, Tranel D, Adolphs R. Evidence for preserved emotional memory in normal older persons. Emotion. 2003;3(3):239-53.

102. Pitkänen A, Savander V, LeDoux JE. Organization of intra-amygdaloid circuitries in the rat: an emerging framework for understanding functions of the amygdala. Trends Neurosci.

1997;20(11):517-23.

103. Roozendaal B, McGaugh JL. Amygdaloid nuclei lesions differentially affect glucocorticoidinduced memory enhancement in an inhibitory avoidance task. Neurobiol Learn Mem.

1996;65(1): 1-8.

104. Roozendaal B, McGaugh J L. Basolateral amygdala lesions block the memory-enhancing effect of glucocorticoid administration in the dorsal hippocampus of rats. Eur J Neurosci. 1997;9(1):76-83.

105. Gewirtz JC, Davis M. Second-order fear conditioning prevented by blocking NMDA receptors in amygdala. Nature. 1997;388(6641):471-4.

106. Swanson LW, Petrovich GD. What is the amygdala? Trends Neurosci. 1998;21(8):323-31.

107. Gallagher M, Chiba AA. The amygdala and emotion. Curr Opin Neurobiol. 1996;6(2):221-7.

108. Killcross S, Robbins TW, Everitt BJ. Different types of fear-conditioned behaviour mediated by separate nuclei within amygdala. Nature. 1997;388(6640):377-80.

109. Packard MG, Teather LA. Amygdala modulation of multiple memory systems: hippocampus and caudate-putamen. Neurobiol Learn Mem. 1998;69(2): 163-203.

110. McGaugh J L. The amygdala modulates the consolidation of memories of emotionally arousing experiences. Ann Rev Neurosci. 2004;27:1-28.

111. Blanchard DC, Blanchard RJ. Innate and conditioned reactions to threat in rats with amygdaloid lesions. J Comp Physiol Psychol. 1972;81(2):281-90.

112. Canteras NS, Ribeiro-Barbosa ER, Comoli E. Tracing from the dorsal premammillary nucleus prosencephalic systems involved in the organization of innate fear responses. Neurosci Biobehav Rev. 2001;25(7-8):661-8.

113. LeDoux JE, Cicchetti P, Xagoraris A, Romanski LM. The lateral amygdaloid nucleus: sensory interface of the amygdala in fear conditioning. J Neurosci. 1990; 10(4): 1062-9.

114. Davis M. The role of the amygdala in emotional learning. Int Rev Neurobiol. 1994;36:225-66.

115. Maren S, Fanselow MS. The amygdala and fear conditioning: has the nut been cracked? Neuron. 1996; 16(2): 237-40.

116. Muller J, Corodimas KP, Fridel Z, LeDoux JE. Functional inactivation of the lateral and basal nuclei of the amygdala by muscimol infusion prevents fear conditioning to an explicit conditioned 
stimulus and to contextual stimuli. Behav Neurosci. 1997;111(4):683-91.

117. Quirk GJ, Armony JL, LeDoux JE. Fear conditioning enhances different temporal components of tone-evoked spike trains in auditory cortex and lateral amygdala. Neuron. 1997;19(3):613-24.

118. Wilensky AE, Schafe GE, LeDoux JE. Functional inactivation of the amygdala before but not after auditory fear conditioning prevents memory formation. J Neurosci. 1999; 19(24): RC48.

119. Fanselow MS, LeDoux JE. Why we think plasticity underlying Pavlovian fear conditioning occurs in the basolateral amygdala. Neuron. 1999;23(2):229-32.

120. Clugnet MC, LeDoux JE. Synaptic plasticity in fear conditioning circuits: induction of LTP in the lateral nucleus of the amygdala by stimulation of the medial geniculate body. J Neurosci. 1990;10(8): 2818-24.

121. Rogan MT, Staubli UV, LeDoux JE. Fear conditioning induces associative long-term potentiation in the amygdala. Nature. 1997;390(6660):604-7.

122. Maren S, Fanselow MS. Synaptic plasticity in the basolateral amygdala induced by hippocampal formation stimulation in vivo. J Neurosci. 1995; 15(11):7548-64.

123. Schroeder BW, Shinnick-Gallagher P. Fear learning induces persistent facilitation of amygdala synaptic transmission. Eur J Neurosci. 2005;22(7):1775-83.

124. Yeh SH, Mao SC, Lin HC, Gean PW. Synaptic expression of glutamate receptor after encoding of fear memory in the rat amygdala. Mol Pharmacol. 2006;69(1):299-308.

125. Shumyatsky GP, Tsvetkov E, Malleret G, Vronskaya S, Hatton M, Hampton L, et al. Identification of a signaling network in lateral nucleus of amygdala important for inhibiting memory specifically related to learned fear. Cell. 2002;111(6):905-18.

126. Applegate CD, Frysinger RC, Kapp BS, Gallagher M. Multiple unit activity recorded from amygdala central nucleus during Pavlovian heart rate conditioning in rabbit. Brain Res.

$1982 ; 238(2): 457-62$.

127. Pascoe JP, Kapp BS. Electrophysiological characteristics of amygdaloid central nucleus neurons during Pavlovian fear conditioning in the rabbit. Behav Brain Res. 1985; 16(2-3): 117-33.

128. Maren S, Yap SA, Goosens KA. The amygdala is essential for the development of neuronal plasticity in the medial geniculate nucleus during auditory fear conditioning in rats. J Neurosci. 2001;21(6): RC135.

129. Goosens KA, Maren S. NMDA receptors are essential for the acquisition, but not expression, of conditional fear and associative spike firing in the lateral amygdala. Eur J Neurosci.

2004;20(2):537-48.

130. Naylor JC, Simson PE, Gibson B, Schneider AM, Wilkins E, Firestone A, et al. Ethanol inhibits spontaneous activity of central nucleus of the amygdala neurons but does not impair retention in the passive-avoidance task. Alcohol Clin Exp Res. 2001;25(11):1683-8.

131. Collins DR, Pelletier JG, Pare D. Slow and fast (gamma) neuronal oscillations in the perirhinal cortex andlateral amygdala. J Neurophysiol. 2001;85(4):1661-72.

132. Schoenbaum G, Setlow B, Ramus SJ. A systems approach to orbitofrontal cortex function: recordings in ratorbitofrontal cortex reveal interactions with different learning systems. Behav Brain Res. 2003; 146(1-2): 19-29.

133. Liang KC, McGaugh JL, Martinez JL J r, J ensen RA, Vasquez BJ, Messing RB. Post-training amygdaloid lesions impair retention of an inhibitory avoidance response. Behav Brain Res. $1982 ; 4(3): 237-49$.

134. Parent MB, Quirarte GL, Cahill L, McGaugh JL. Spared retention of inhibitory avoidance 
learning after posttraining amygdala lesions. Behav Neurosci. 1995; 109(4): 803-7.

135. McGaugh J L, Cahill L, Roozendaal B. Involvement of the amygdala in memory storage: interaction with other brain systems. Proc Natl Acad Sci USA. 1996; 93(24): 13508-14.

136. Vazdarjanova A, McGaugh J L. Basolateral amygdala is involved in modulating consolidation of memory for classical fear conditioning. J Neurosci. 1999;19(15):6615-22.

137. Vazdarjanova A, McGaugh J L. Basolateral amygdala is not critical for cognitive memory of contextual fear conditioning. Proc Natl Acad Sci USA. 1998; 95(25):15003-7.

138. Cahill L, Weinberger NM, Roozendaal B, McGaugh J L. Is the amygdala a locus of "conditioned fear"? Some questions and caveats. Neuron. 1999;23(2):227-8.

139. Lorenzini CA, Baldi E, Bucherelli C, Tassoni G. Time-dependent deficits of rats memory consolidation induced by tetrodotoxina injections into the caudate-putamen, nucleus accumbens, and globus pallidus. Neurobiol Learn Mem. 1995;63(1):87-93.

140. Burns LH, Annett L, Kelley AE, Everitt BJ, Robbins TW. Effects of lesions to amygdala, ventral subiculum, medial prefrontal cortex, and nucleus accumbens on the reaction to novelty: implication for limbic-striatal interactions. Behav Neurosci. 1996; 110(1):60-73.

141. Kemble ED, Blanchard DC, Blanchard RJ. Effects of regional amygdaloid lesions on flight and defensive behaviors of wild black rats (Rattus rattus). Physiol Behav. 1990;48(1): 1-5.

142. Sanders SK, Shekhar A. Regulation of anxiety by GABAA receptors in the rat amygdala. Pharmacol Biochem Behav. 1995;52(4):701-6.

143. Bellgowan PS, Helmstetter FJ. Neural systems for the expression of hypoalgesia during nonassociative fear. Behav Neurosci. 1996;110(4):727-36.

144. Mesches MH, Bianchin M, McGaugh J L. The effects of intra-amygdala infusion of the AMPA receptor antagonist CNQX on retention performance following aversive training. Neurobiol Learn Mem. 1996; 66(3): 324-40.

145. Walker DL, Davis M. Double dissociation between the involvement of the bed nucleus of the stria terminalis and the central nucleus of the amygdala in startle increases produced by conditioned versus unconditioned fear. J Neurosci. 1997; 17(23): 9375-83.

146. Antoniadis EA, McDonald RJ. Amygdala, hippocampus, and unconditioned fear. Exp Brain Res. $2001 ; 138(2): 200-9$.

147. Squire LR, Knowlton B, Musen G. The structure and organization of memory. Ann Rev Psychol. 1993;44:453-95.

148. Weiskrantz L, Warrington EK. Conditioning in amnesic patients. Neuropsychologia. 1979; 17(2): 187-94.

149. Roozendaal B, Portillo-Marquez G, McGaugh JL. Basolateral amygdala lesions block glucocorticoid-induced modulation of memory for spatial learning. Behav Neurosci.

1996;110(5): 1074-83.

150. Roozendaal B, McReynolds JR, McGaugh J L. The basolateral amygdala interacts with the medial prefrontal cortex in regulating glucocorticoid effects on working memory impairment. J Neurosci. 2004;24(6): 1385-92.

151. Cahill L. Neurobiological mechanisms of emotionally influenced, long-term memory. Prog Brain Res. 2000; 126:29-37.

152. Miranda MI, McGaugh JL. Enhancement of inhibitory avoidance and conditioned taste aversion memory with insular cortex infusions of 8-Br-cAMP: involvement of the basolateral amygdala. Learn Mem. 2004; 11(3):312-7. 
153. LaLumiere RT, Nawar EM, McGaugh JL. Modulation of memory consolidation by the basolateral amygdala or nucleus accumbens shell requires concurrent dopamine receptor activation in both brain regions. Learn Mem. 2005;12(3):296-301.

154. Mclntyre CK, Miyashita T, Setlow B, Marjon KD, Steward O, Guzowski JF, et al. Memoryinfluencing intra-basolateral amygdala drug infusions modulate expression of Arc protein in the hippocampus. Proc Natl Acad Sci USA. 2005; 102(30): 10718-23.

155. Malin EL, McGaugh J L. Differential involvement of the hippocampus, anterior cingulate cortex, and basolateral amygdala in memory for context and footshock. Proc Natl Acad Sci USA. 2006; 103(6): 1959-63.

156. LaLumiere RT, Buen TV, McGaugh JL. Post-training intra-basolateral amygdala infusions of norepinephrine enhance consolidation of memory for contextual fear conditioning. J Neurosci. 2003;23(17):6754-8.

157. Shumyatsky GP, Malleret G, Shin RM, Takizawa S, Tully K, Tsvetkov E, et al. Stathmin, a gene enriched in the amygdala, controls both learned and innate fear. Cell. 2005; 123(4):697-709.

158. Muller M, Fendt M. Temporary inactivation of the medial and basolateral amygdala differentially affects TMT-induced fear behavior in rats. Behav Brain Res. 2006;167(1):57-62.

159. Canteras NS. Critical analysis of the neural systems organizing innate fear responses. Rev Bras Psiquiatr. 2003;25(Suppl 2):21-4.

\section{ن Correspondência}

Regina H. Silva

Centro de Biociências, Universidade Federal do Rio Grande do Norte (UFRN)

Av. Salgado Filho, $s / n$

Caixa Postal 1511, CEP 59078-970, Natal, RN

Fax: (84) 3211.9206

E-mail: reginasilva@cb.ufrn.br

Recebido em 10/08/2007.

Aceito em 16/11/2007.

Suporte Financeiro: Conselho Nacional de Desenvolvimento Científico e Tecnológico (CNPq) (305029/2004-5 e 472625/2006-3) e Coordenação de Aperfeiçoamento de Pessoal de Nível Superior (CAPES) (PROAP/UFRN).

Não foram declarados conflitos de interesse associados à publicação deste artigo. 